\title{
Paraplegia
}

\section{Use of Plasmid Analysis to Determine the Source of Bacterial Invasion of the Urinary Tract}

\author{
W. H. Donovan, MD, R. Hull, PhD, D. X. Cifu, MD, H. D. Brown, PhD, \\ N. J. Smith, MT \\ The Institute for Rehabilitation and Research and Baylor College of Medicine, \\ Houston, Texas, USA.
}

\section{Summary}

Gram negative colonisation and infection of the urinary tract is a well recognised complication of the neuropathic bladder caused by spinal cord injury (SCI). K. pneumoniae accounts for one third of all urinary tract infections in hospitalised SCI patients. Plasmid analysis has been shown to reliably fingerprint bacterial strains, particularly $\mathrm{K}$. pneumoniae, so that growth from two separate locations in or on the body can be accurately analysed as to migration from a reservoir to a target location.

Eighty seven hospitalised SCI patients on intermittent catheterisation for a total of 586 patient-weeks were studied. Twice weekly catheterised urine specimens and once weekly rectal swab cultures were taken from each patient. Thirty seven patients experienced at least one clinically significant (colony count $>10$ 000/mL) urinary tract colonisation caused by K. pneumoniae, representing 66 total colonisations. Further analysis of 31 of these 37 patients revealed: $\mathrm{K}$. pneumoniae in all of their stool cultures $(p<0.05)$ and the identical strain of $\mathrm{K}$. pneumoniae in the urine as well as the stool in $72 \%$ of the 66 colonisations $(p<0.05)$. Analysis of 14 patients without $\mathrm{K}$. pneumoniae urinary colonisations showed absence of faecal $\mathrm{K}$. pneumoniae in 3, and predominant growth in only 4 . In 22 of the 37 patients, multiple $\mathrm{K}$. pneumoniae urinary colonisations were noted, representing 27 pairs of colonisation. Fifteen of the pairs were found to be relapsing (caused by two identical bacterial strains), and 12 were recurrent (caused by two different bacterial strains). Thirteen of the 15 relapsing pairs also had identical urine and stool $\mathrm{K}$. pneumoniae strains $(p<0.05)$. All colonisations were treated with appropriate antibiotics based on culture and sensitivity reports. Fourteen of the 15 relapsing colonisation pairs have identical antibiograms $(p<0.05)$, while all 12 of the recurrent colonisation pairs had different antibiograms $(p<0.05)$. The differences noted on sensitivity patterns (antibiograms) correlated with differences among strains of $\mathrm{K}$. pneumoniae based upon plasmid analysis. Treatment of bacteriuria did not affect the nature of repeated colonisations regardless of the antibiotic chosen, the route of administration or the duration of treatment. 
We conclude that $\mathrm{K}$. pneumoniae found in the urinary tract of spinal cord patients usually derive from that individual's own bowel flora, particularly in the case of relapsing bacteriuria. Further, relapsing bacteriuria in patients on intermittent catheterisation is typically not due to urinary tract lithiasis or other urinary tract pathology. These results also suggest that abundant bowel colonisation with $\mathrm{K}$. pneumoniae is a predisposing but not a prerequisite factor for subsequent urinary colonisation. The clinical and epidemiological importance of this data warrants further study.

Key words: Spinal Cord Injury; Bacteriuria; Plasmid analysis; Epidemiology.

Urinary tract infections (UTI's) are a major cause of morbidity in spinal cord injury (SCI) patients and, since repeated episodes of bacteriuria may contribute to the deterioration of kidney function, they also represent a risk factor regarding mortality [Brown, 1982-1983; Donovan, 1978; Erickson, 1982; Rennie, 1978]. Twenty years ago the urological prognosis for most SCI patients was one of constant or repeated bacteriuria, with episodes of symptomatic UTI's being common. The first stages of pyelonephritis in some patients began prior to the end of the initial rehabilitation period. Improvements and changes in the medical and surgical management of SCI patients in recent years have substantially decreased the risk of patients developing multiple UTI's and the consequences of recurrent pyelonephritis. The expanded use of intermittent catheterisation programs (ICP) have helped to maintain sterile urine, in addition to reducing the incidence of bladder calculi and complications of the male genitalia. Early detection and amelioration of severe detrusor sphincter dyssynergia has decreased the incidence of hydronephrosis, vesicoureteral reflux and autonomic dysreflexia. Regular annual follow-up has revealed impending complications in early stages, before extensive tissue damage can occur. As these developments occurred, there has been a concomitant transition in the attitudes of rehabilitation physicians and scientists in regard to the practicality of maintaining SCI patients in a bacteriuria-free state during the rehabilitation process and on into the return to the community. This transition is evidenced by the use of therapeutic and prophylactic regimens to keep these patients abacteriuric and, by reports in the literature of studies directed towards understanding the epidemiology of UTI's in SCI patients [Anderson, 1983; Donovan, 1978; Erickson, 1982; Krebs, 1984; Montgomerie, 1979]. This area of knowledge is not well developed [Casewell, 1978; Christensen, 1972; Cooke and Brayson, 1979].

National Spinal Cord Injury Data Research Center statistics reveal that there are over 7000 new SCI patients annually and over $65 \%$ of all SCI patients experience at least one UTI during their initial rehabilitation period [Young, 1982]. In rehabilitation hospitals $K$. pneumoniae accounts for 25 to $36 \%$ of all UTI's in SCI patients [Brown, 1982-1984; Donovan, 1978; Krebs, 1984]. Body colonisation of hospitalised patients with $K$. pneumoniae is well documented [Bierman, 1951; Casewell, 1978; Davis, 1974; Gilmore, 1982; Holzman, 1974; Jonsson, 1973; Montgomerie, 1979; Montgomerie, 1970; Montgomerie, 1983; Pollack, 1972; Rose, 1968]. Factors common to many SCI patients, i.e. ICU care, surgery with anaesthesia, urinary catheterisation, and antimicrobial therapy, are known to promote bowel colonisation by K. pneumoniae [Bierman, 1951; Gilmore, 1982; Houang, 1980; 
Maki, 1978; Montgomerie, 1979; Montgomerie, 1970; Noriega, 1975; Pollack, 1972; Rose, 1968]. The epidemiological relationship between bowel colonisation and the development of urinary tract infections caused by $K$. pneumoniae is not well understood [Cooke and Brayson, 1979; Montgomerie, 1979; Selden, 1971]. The development of effective strategies for the nosocomial management of urinary pathogens, such as $K$. pneumoniae, depends on a highly precise understanding of the colonisation/infection interaction that is not achievable with conventional epidemiological study methods [Casewell, 1975; Casewell, 1981; Cooke and Brayson, 1979; De Silva, 1977; Hessek, 1981; Markowitz, 1980; Portnoy, 1981; Rennie, 1974; Rennie, 1978; Riser, 1981; Simmons-Smit, 1983; Smith, 1982; Tietze, 1983]. Therefore, a process known as plasmid DNA analysis has been utilised to study this interaction.

Plasmids are auxiliary circular DNA molecules found in many bacterial species, and can vary in size and number in different bacterial strains. The plasmid content of a bacterium is relatively stable and, because of the many possible size-number combinations, the plasmid pattern is essentially unique for each unrelated isolate. Plasmid DNA analysis can therefore provide a unique 'fingerprint' for each strain of bacterium found among a group of bacteria. K. pneumoniae is an organism particularly well suited to plasmid DNA analysis because of the frequency with which plasmids are found and the ease with which they can be separated. Successful epidemiologic studies investigating Klebsiella and Salmonella outbreaks have been completed employing plasmid analysis [Holmberg, 1981; John, 1983; Taylor, 1982].

This investigation utilised plasmid DNA analysis, in conjunction with conventional epidemiological techniques to provide information on the role of $K$. pneumoniae in the spinal cord injured patient, specifically exploring; the role of a faecal reservoir for subsequent urinary colonisations, the incidence of same or different bacterial strains in repeated urinary colonisations, and the efficacy of antibiotic therapy in repeated urinary colonisations.

\section{Materials and methods}

Subjects

From January 1986 to December 1989, 87 newly injured spinal cord injured patients were admitted for inpatient rehabilitation and were expected to be maintained on intermittent catheterisation for at least 6 weeks. There were 16 females (18\%) and 71 males (82\%), 33 paraplegics (38\%) and 54 quadriplegics (62\%). The ages ranged from 16 to 66 years, with a median age of 27 years (see Table I).

The patients were studied from 1 to 19 weeks, with a mean duration of $6 \cdot 8$ weeks. Total patient weeks of the study was 586 weeks.

\section{Data collection}

Rectal swabs were collected within 24 hours of enrollment in the program. These were repeated weekly (Monday) thereafter. The swabs were plated on McConkey media for identification of the predominant organism by routine microbiological 
Table I Demographics

\begin{tabular}{ll}
\hline \multicolumn{2}{l}{ Total patients 87} \\
Sex \\
$\begin{array}{l}\text { females } \\
\text { males }\end{array}$ & $16(18 \%)$ \\
SCI $\quad 71(82 \%)$ \\
$\quad$ paraplegic \\
quadriplegic & $33(38 \%)$ \\
\hline
\end{tabular}

laboratory techniques. Additionally, the swabs were plated on Klebsiella selective media. If growth occurred, the identification was confirmed and 10 isolates were picked and stocked for later analysis. Prior to this study $50 \mathrm{~K}$. pneumoniae colonies were sampled and subjected to plasmid analysis. It was determined using standard probability tables that by sampling 10 colonies from the Klebsiella specific media, one would have between a $65 \%$ to $99 \%$ probability of collecting all strains of $K$. pneumoniae present in the stool.

Sterile, catheterised urine specimens were obtained for culture within 24 hours of enrollment. These were repeated twice weekly (Monday and Thursday) thereafter. All intermittent catheterisations were performed 4 to 6 times daily, and all were collected using a sterile technique (sterile gloves, catheterisation kits). The urine specimens were plated on blood agar, and subsequent bacterial growth was identified in the conventional manner. If Klebsiella were identified, 10 colonies were picked and stocked for later analysis.

If the urine cultures were positive for $K$. pneumoniae ( $>10000$ colonies $/ \mathrm{ml}$ ), then the stool isolates from 1 or 2 weeks prior to the positive urine culture and the urine isolate were prepared for plasmid analysis.

Patients with two or more positive urine cultures greater than 2 weeks apart, with a negative culture in between, also had their urine isolates prepared for plasmid analysis.

Finally, a record of the antibiotic regimens employed in all patients was made, and subsequent repeat urine colonisations noted.

The plasmid DNA purification technique was performed on the appropriate culture specimens prior to analysis. The following steps were followed:

1. $2 \mathrm{ml}$ of culture broth was grown overnight.

2. Bacteria were harvested by centrifugation and treated with alkaline detergent lytic solution to lyse bacteria and denature the DNA.

3. This solution was then neutralised with TRIS to restore plasmid DNA to its double stranded structure (i.e. renature the DNA).

4. Chromosomal DNA was then selectively precipitated, removed by centrifugation and discarded.

5. The remaining plasmid DNA was precipitated and collected by centrifugation.

6. The plasmid DNA was resuspended in buffer for agarose gel electrophoresis.

7. Electrophoresis patterns were then analysed to identify isolates.

Details of this method are presented elsewhere (Portnoy, 1972). 


\section{Results}

\section{Urine/stool colonisation}

$K$. pneumoniae was found in the urine of 37 patients (43\%) at least once during the study period. Of these 37 patients, 15 (41\%) were colonised once and 22 (59\%) were colonised more than once, representing 66 total colonisations. This represented one colonisation per each 8.9 patient weeks, or 0.1 colonisations per week (Table II).

Thirty one of these 37 patients were studied further. Their stool was analysed coinciding with the time their urine was colonised to determine if $K$. pneumoniae was the predominant organism. In 19 patients $(61 \%)$ it was the predominant organism and in $12(39 \%)$ the growth was sparse. K. pneumoniae was present in the stool of this entire group. Similarly, 14 of the 50 patients who did not grow out $K$. pneumoniae in their urine during the study period and who were hospitalised for at least 6 weeks, to allow for bowel recolonisation, were further studied. In 4 patients (29\%) K. pneumoniae was the predominant organism, in $7(50 \%)$ the growth was sparse, and in $3(21 \%)$ it was not found (Table III). These differences between the 31 who grew out $K$. pneumoniae and the 14 who didn't were statistically significant ( $\mathrm{p}<0.05, \mathrm{z}$-test for significance of a proportion). Twenty-nine of the 37 patients who grew out $K$. pneumoniae in their urine, representing 44 colonisations, had their urine and stool strains compared by plasmid DNA analysis. Thirty one colonisations $(70 \%)$ of the 44 had identical urine and stool strains, while 13 colonisations $(30 \%)$ had different strains. These results were statistically significant $(p<0.05$,

Table II $K$. pneumoniae urine colonisations

\begin{tabular}{cc}
\hline Patients with positive cultures $(>10000 \mathrm{col} / \mathrm{ml})$ \\
One colonisation & $15(17 \%)$ \\
Two colonisations & $15(17 \%)$ \\
Three colonisations & $7(8 \%)$ \\
& $37(43 \%)$
\end{tabular}

Patients without positive cultures

$$
\begin{aligned}
& 50(57 \%) \\
& 87(100 \%)
\end{aligned}
$$

\begin{tabular}{|c|c|c|c|}
\hline & Predominant & Sparse & None \\
\hline $\begin{array}{l}\text { Urinary } K . \text { pneumoniae } \\
\text { Present ( } 31 \text { patients) }\end{array}$ & $19(61 \%)$ & $12(39 \%)$ & $0(0 \%)$ \\
\hline $\begin{array}{l}\text { Urinary } K . \text { pneumoniae } \\
\text { Absent ( } 14 \text { patients) }\end{array}$ & $4(29 \%)$ & $7(50 \%)$ & $3(21 \%)$ \\
\hline
\end{tabular}

Table III $K$. pneumoniae growth from stool 
$z$-test), and support the premise that the stool serves as a reservoir of $K$. pneumoniae for subsequent colonisation of the urinary tract in SCI patients.

\section{Repeated urinary colonisation}

As mentioned earlier, 22 (59\%) of the 37 patients with $K$. pneumoniae urinary colonisation had 2 or more colonisations. Plasmid analysis was available for 17 of these patients. Twelve had 2 colonisations, representing 12 pairs, and 5 had 3 colonisations, representing 15 pairs, for a total of 27 pairs of colonisations (Table IV). The plasmid DNA of these 27 pairs of repeated $K$. pneumoniae colonisations were compared to distinguish between relapsing (identical bacterial strains) and recurrent (different bacterial strains) colonisations. Fifteen of the pairs $(56 \%)$ were found to be relapsing and 12 of the pairs (44\%) were found to be recurrent colonisations.

A comparison of the plasmid DNA analysis of the 15 pairs of relapsing colonisation and their respective stool cultures, revealed that 13 of the $15(87 \%)$ had identical urine and bowel strains. This relationship was statistically significant $(\mathrm{p}<0.05, \mathrm{z}$-test) and would suggest that the stool serves as a reservoir for the vast majority of relapsing bacteriuria.

The treatment of the initial colonisation of these 27 pairs was examined, both for the type and duration of antibiotic treatment. The average period of treatment for repeated colonisations which proved to be relapsing was 11.6 days (range 7 to 21 days) and for colonisations which proved to be recurrent was 12.3 days (range 6 to 21 days). This difference was not statistically significant. Additionally, no statistically significant trends of the class of antibiotic used or the method of administration could be discerned (Tables $\mathrm{V}$ and VI).

The standard laboratory antibiograms from the urine cultures of these 27 pairs of colonisations were also reviewed. Fourteen of the 15 pairs $(93 \%)$ of relapsing colon-

Table IV Repeated $K$. pneumoniae urine colonisations

12 patients with 2 colonisations $(\mathrm{A}, \mathrm{B})$ :

$$
\mathrm{A} \longrightarrow \mathrm{B}=12 \text { pairs }
$$

5 patients with 3 colonisations $(\mathrm{A}, \mathrm{B}, \mathrm{C})$ :

$$
\begin{aligned}
& \mathrm{A} \longrightarrow \mathrm{B} \\
& \mathrm{A} \longrightarrow \mathrm{C} \\
& \mathrm{B} \longrightarrow \mathrm{C}=15 \text { pairs }
\end{aligned}
$$

27 pairs

Table $\mathbf{V}$ Antibiotic treatment of urinary colonisation (duration and route of administration)

\begin{tabular}{lccc}
\hline & $\begin{array}{c}\text { Length of } \\
\text { treatment }\end{array}$ & $\begin{array}{c}\text { Oral } \\
\text { dosing }\end{array}$ & $\begin{array}{c}\text { Intravenous } \\
\text { dosing }\end{array}$ \\
$\begin{array}{l}\text { Relapsing colonisation } \\
\text { Recurrent colonisation }\end{array}$ & 11.6 days & $>95 \%$ & $<5 \%$ \\
\hline
\end{tabular}


Table VI Antibiotic treatment of urinary colonisation (per cent of urinary colonisations treated with each antibiotic class $)^{\star}$

\begin{tabular}{lcc}
\hline Type & $\begin{array}{c}\text { Relapsing } \\
\text { colonisation }\end{array}$ & $\begin{array}{c}\text { Recurrent } \\
\text { colonisation }\end{array}$ \\
\hline Nitrofurantoin & $53 \%$ & $67 \%$ \\
Cephalosporin & $40 \%$ & $33 \%$ \\
Fluoroquinolone & $40 \%$ & $33 \%$ \\
Sulfonamide & $33 \%$ & $50 \%$ \\
Penicillin & $20 \%$ & $25 \%$ \\
Aminoglycoside & $13 \%$ & $17 \%$ \\
Tetracyline & $7 \%$ & $8 \%$ \\
\hline
\end{tabular}

^Totals are greater than $100 \%$ because many colonisations were treated with more than one antibiotic, because: (a) some patients were on prophylactic doses of one then started on another: (b) changes were made when sensitivities returned or (c) cultures from other sources required treatment with a second antibiotic

isations (identical K. pneumoniae strain) had identical antibiograms, while 12 of 12 pairs $(100 \%)$ of recurrent colonisations (different $K$. pneumoniae strains) had different antibiograms. These findings were statistically significant $(p<0.05, z$-test $)$ and support the view that the antibiogram is generally an accurate measure of determining different strains of the same species of $K$. pneumoniae.

Owing to the relatively small number of female patients (16), observations concerning male-female differences were not statistically valid.

\section{Discussion}

The goal of this investigation was to provide a better understanding of the epidemiology of $K$. pneumoniae in the SCI patient, and to explore the clinical applications of specialised epidemiological tools. Plasmid DNA analysis has provided a very reliable and relatively simple means of achieving these goals. Prior studies have shown $K$. pneumoniae to be a common bowel coloniser in SCI patients, however the relationship between bowel and urine colonisation was poorly understood [Bierman, 1951; Cooke and Brayson, 1979; Cooke and Pool, 1979; Gilmore, 1982; Houang, 1980; Maki, 1978; Montgomerie, 1979; Montgomerie, 1970; Noriega, 1975; Pollack, 1972; Rose, 1968; Selden, 1971]. The data presented (Table III) would suggest that the stool is most likely the primary source of urinary $K$. pneumoniae, and in episodes of relapsing bacteriuria this relationship is especially true. Additionally, a predominance of $K$. pneumoniae seems to be a predisposing but not a prerequisite factor for subsequent urinary growth. The proximity of the perineal structures (i.e. male vs. female), the frequency of bowel incontinence, and the appropriateness of technique for bowel and bladder care can all play a vital role in the transmission of bacteria from the bowel reservoir. Despite these and other risk factors, in a setting where principles of cross infection are strictly enforced, the incidence of $K$. pneumoniae urinary colonisation remains low (circa one colonisation for every 9 weeks of hospitalisation or 372 catheterisations). 
Whereas in the past, repeated urinary tract colonisation with the same bacteria had often been presumed to be a relapsing growth, these data demonstrate that over $40 \%$ of these recolonisations by $K$. pneumoniae are by different strains. Therefore the need to undertake extensive investigations to discover a presumed reservoir in the urinary tract may be obviated. Thus, a patient without an obvious source of bacteria, such as renal/bladder stones, prostatitis or urethral/bladder diverticulae, can be spared further evaluation. The same can also be said even when recolonisation is caused by the identical strain previously isolated, since a bowel reservoir can be presumed in most cases of relapsing $K$. pneumoniae colonisation.

No specific antibiotic used to treat a $K$. pneumoniae colonisation was found that seemed to protect against or favour the occurrence of relapsing colonisation. Thus, the choice of antibiotic therapy when prescribed for the durations given in this study, does not seem to play a major role in whether relapsing versus recurrent urinary colonisations occur. More aggressive perineal hygiene and judicious use of urinary prophylaxis (i.e. monitoring its effectiveness in limiting specific colonisations) may reduce the impact that a reservoir in the stool can produce, however. The use of routine antibiogram, if plasmid analysis is not available, to assess the nature of repeated colonisations may provide an efficient means of determining whether they are recurrent or relapsing. However, accuracy of antibiograms may vary among laboratories.

This investigation while providing epidemiological as well as clinically important information has not controlled for a number of factors which may have contributed to the observed results, primarily because of the complexity of the task. These confounding variables include; the varying types of neuropathic bowel/bladder that may occur in SCI patients, the role of external catheters, the use of prophylactic, antibiotics, types of bowel evacuation, frequency of catheterisation, level of SCI, other non-Klebsiella colonisation and the possibility that certain bacterial strains may have been missed secondary to sampling error. Future studies should evaluate these areas further.

\section{Conclusions}

The epidemiology of bacterial colonisation of the stool and urine of SCI patients is extremely complex. Plasmid DNA analysis and careful microbiological examination provide an excellent means of assessing the relationship between transmission of a bacterium from a reservoir to a target area. $K$. pneumoniae is readily studied with these techniques, and appears to be spread from the faeces to the urine in most SCI patients undergoing intermittent catheterisation. Even episodes of repeat urinary colonisation with the same strain of bacterium (relapsing) seems to be often directly related to a bowel reservoir. Therefore, when no cause for relapsing bacteriuria can be found in the GU tract, patients may be safely treated expectantly as symptoms warrant since the source is probably from the bowel. The length, route or specific class of antibiotic treatment do not appear to be a major determining factor in the spread of this organism. Antibiograms may provide a reliable means of evaluating the nature (i.e. the same versus a different strain of a particular species) of repeated bacterial colonisation since they correlate extremely well with strain differentiation by plasmid analysis. Further research in this area is vital. 


\section{References}

ANDERSON RU, HSIEH-MA ST 1983 Association of bacteriuria and pyuria during intermittent catheterization after spinal cord injury. Fournal of Urology 130:299-301.

BIERMAN HR, JAWETZ E 1951 The effect of prolonged administration of antibiotics on the human fecal flora. Fournal of Laboratory and Clinical Medicine 37:394-401.

BROWN H 1982-1984 Nosocomial infection report. The Institute for Rehabilitation and Research.

CASEWELL MW 1975 Titers and cross reactions of commercial antisera for capsular typing of Klebsiella species. Fournal of Clinical Pathology 28:33-36.

CASEWEll MW, Phillips I 1978 Epidemiological patterns of Klebsiella colonization and infection in an intensive care ward. F Hyg Camb 80:295-300.

Casewell MW, Phillips I 1981 Aspects of plasmid mediated resistance and epidemiology of Klebsiella species. American fournal of Medicine 70:459-462.

Christensen SC, KoRner B 1972 An endemic caused by multiresistant Klebsiella in an urological unit. Scandinavian fournal of Urology and Nephrology 6:232-238.

Cooke EM, Brayson JC, Edmonson AS, Hall D 1979 An investigation into the incidence and sources of Klebsiella infections in hospital patients. Fournal of Hygiene 82:473-480.

Cooke EM, Pool R, Brayson JC, Edmonson AS, Munro ME, Shinebaum R 1979 Further studies on the sources of Klebsiella aerogenes in hospital patients. Fournal of Hygiene 83:391-395.

DAvis TJ, MATSEN JM 1974 Prevalence and characteristics of Klebsiella species; Relation to association with a hospital environment. Fournal of Infectious Diseases 130:402-405.

DE Silva MI, RuBIN SJ 1977 Multiple biotypes of Klebsiella pneumoniae in single clinical specimens. Fournal of Clinical Microbiology 5:62-65.

Donovan WH, Stolov WC, Clowers DE, Clowers MR 1978 Bacteriuria during intermittent catheterization following spinal cord injury. Archives of Physical Medicine and Rehabilitation 59:351-357.

ERICKSON RP, MERRITT JL, OPITZ JL, ILSTRUP DM 1982 Bacteriuria during follow-up in patients with spinal cord injury; rates of bacteriuria in various bladder emptying methods. Archives of Physical Medicine and Rehabilitation 63:409-412.

Gilmore DS, SCHICK DG, Montgomerie JZ 1982 Psuedomonas aeruginosa and Klebsiella pneumoniae on the perinea of males with spinal cord injuries. Fournal of Clinical Microbiology 16:865-867.

Hessek AP, Peters G, Byczynska B, Pulverer G 1981 Phage-typing of Klebsiella strains from Cologne and Wroclaw. Zentralblatt fur Bakteriologie und Hygiene 250:296-306.

Holmberg SD, WaChSMUth IK, Hickman-Brenner FW, COHEN ML 1981 Comparison of plasmid profile analysis, phage typing and antimicrobial susceptibility testing in characterizing Salmonella typhimurium isolates from outbreaks. Fournal of Clinical Microbiology 19:100-104.

Holzman RS, Florman AL, Podrid PJ, Simbrkoff MS, Toharsky B 1974 Drug associated diarrhea as a potential reservoir for hospital infections. Lancet i:1195-1196.

Houang ET, Casewell MW, Simms PA, Horton RA 1980 Hospital acquired fecal Klebsiella as a source of multiple resistance in the community. Lancet $\mathrm{i}: 148-149$.

JoHN JF, MCKEE KT, TwitTy JA, SCHAFFNER W 1983 Molecular epidemiology of sequential nursery epidemics caused by multiresistant Klebsiella pneumoniae. Fournal of Pediatrics 102:825-830.

JoNSSON M 1973 Antibiotic resistance and R factors in gram negative bacteria isolated in a hospital for infectious diseases. Scandinavian fournal of Infectious Diseases 5:49-54.

Krebs M, Halvorsen RB, Fishman IJ, Santos-Mendoza N 1984 Prevention of urinary tract infection during intermittent catheterization. Fournal of Urology 131:82-86.

MAKI DG 1978 Control of colonization and transmission of pathogenic bacteria in the hospital. Annals of Internal Medicine 89:777-780.

Markowitz SM, Vaezey JM, MaCrina FL, Mayhall CG, LAmb VA 1980 Sequential outbreaks of infection due to Klebsiella pneumoniae in a neonatal intensive care unit: Implication of a conjugative $\mathrm{R}$ plasmid. Fournal of Infectious Diseases 142:106-112.

MONTGOMERIE JZ 1979 Epidemiology of Klebsiella and hospital-associated infections. Reviews of Infectious Diseases 1:736-753.

MONTGOMERIE JZ, DOAK PB, TAYLOR DEM, NorTh JDK 1970 Klebsiella in fecal flora of renal transplant patients. Lancet i:787-791.

Montgomerie JZ, SCHICK DG, Gilmore DS, Graham I $1983 \mathrm{pH}$ and water content of Pseudomonas aeruginosa and Klebsiella pneumoniae colonized perineal skin of men with spinal cord injuries. Fournal of Clinical Microbiology 18:844-848.

Noriega ER, Leibowitz RE, Richmond AS, Rubinstein E, Schaefler S, Simberkoff MW, RAHAL JJ 1975 Nosocomial infection caused by gentamicin-resistant, streptomycin-sensitive Klebsiella. Fournal of Infectious Diseases 131:S45-S50.

Pollock M, Nieman RE, Reinhardt JA, Charache P, Jett MP, Hardy PH 1972 Factors influencing colonization and antibiotic resistance patterns of gram negative bacteria in hospital patients. Lancet ii:668-671. 
Portnoy DA, Moseley SL, Falkow S 1974 Characterization of plasmids and plasmid-associated determinants of Yersinia enterocolitica pathogenesis. Infection and Immunity 31:775-782.

RENNIE RP, DUNCAN IBR 1974 Combined biochemical and serological typing of clinical isolates of Klebsiella. Applied Microbiology 28:534-539.

RENNIE RP, NORD CE, SJOBERG L, DUNCAN IBR 1978 Comparison of bacteriophage typing, serotyping and biotyping as aids in epidemiological surviellance of Klebsiella infections. Fournal of Clinical Microbiology 8:638-642.

RISER E, NoONE P 1981 Klebsiella capsular type versus site of isolatation. Fournal of Clinical Pathology 34:552-555.

Rose HD, SCHREIER J 1968 The effect of hospitalization and antibiotic therapy on the gram negative fecal flora. American fournal of Medical Science 255:228-236.

Selden R, Lee S, Wang WLL, Bennet JV, Eickhoff T 1971 Nosocomial Klebsiella infections; intestinal colonization as a reservoir. Annals of Internal Medicine 74:657-664.

Simmons-Smit AM, Verweij-VAN VUGHT JJ, KANis IYR, MACLAREN DM 1983 Comparison of different methods for bacterial typing of Klebsiella strains. Fournal of Hygiene 90:461-473.

SMITH SM, DIGORI JT, ENG RH 1982 Epidemiology of Klebsiella antibiotic resistance and serotypes. Fournal of Clinical Microbiology 16:868-873.

TAYLOR DN, WACHSMITH IK, SHANGKUAN Y et al 1982 Salmonellosis associated with marijuana; a multistate outbreak traced by plasmid finger printing. New England Fournal of Medicine 306: 1249-1253.

Tietze E, TsChape H 1983 Plasmid pattern analysis of natural bacterial isolates and its epidemiological implication. 7 Hyg Camb 90:475-488.

Young JS, BuRns PE, BowEn AM, MCCUTChEn R 1982 Spinal cord injury statistics experience of the regional spinal cord injury system. Good Samaritan Medical Center, Phoenix, AZ. 